\title{
Single-shot wakefield measurement system
}

\author{
Qiang Gao, Jiaru Shi, and Huaibi Chen \\ Department of Engineering Physics, Tsnghua University, Beijing 100084, People's Republic of China \\ and Key Laboratory of Particle and Radiation Imaging, Tsinghua University, Ministry of Education, \\ Beijing 100084, People's Republic of China \\ Gwanghui Ha \\ POSTECH, Pohang, Gyeongbuk 37673, Republic of Korea \\ John G. Power, Manoel Conde, and Wei Gai \\ Argonne National Laboratory, Lemont, Illinois 60439, USA
}

(Received 4 March 2018; published 26 June 2018)

\begin{abstract}
A new experimental technique for the accurate measurements of wakefields is presented. Knowledge of wakefields is required for many applications ranging from the suppression of wakefields that arise from geometrical discontinuities to the enhancement of wakefields in structure and plasma based wakefield accelerators. In the past, direct measurements of wakefields were done with the drive-witness technique wherein the delay of a short witness beam trailing collinearly behind a high-charge drive beam is scanned over multiple shots and the wakefield is inferred from the energy spectrum of the witness beam. That technique is limited by shot-to-shot jitter, overlap of the drive and witness energy spectrum and the loss of all time-domain information. In this paper, we describe a new technique that overcomes these limitations by measuring the longitudinal phase space of a long witness beam and the drive beam in a single shot. We conclude by presenting experimental results to demonstrate its feasibility using a dielectric structure wakefield accelerator.
\end{abstract}

DOI: 10.1103/PhysRevAccelBeams.21.062801

\section{INTRODUCTION}

Wakefields are an important class of collective effects in many branches of accelerator physics and their accurate measurement is a key to many applications. Wakefields in traditional accelerator beam lines are associated with deleterious effects: beam loading in accelerating structures, beam breakup instability in storage rings, emittance dilution in linear colliders, etc. [1-3]. On the other hand, wakefields are associated with beneficial effects for the development of advanced accelerators: future TeV-scale high energy colliders and compact multiuser free electron laser facilities [4-7]. In both cases, accurate measurement of the wakefield is a key to the success of the application.

Direct measurements of the wakefield have traditionally been done with the drive-witness technique [8-12] wherein a high-charge drive beam is used to excite wakefields and a short, pointlike witness beam is used to probe the wakefield. In this technique, the trailing wakefield is measured

Published by the American Physical Society under the terms of the Creative Commons Attribution 4.0 International license. Further distribution of this work must maintain attribution to the author(s) and the published article's title, journal citation, and DOI. by scanning the delay between the pointlike witness beam and a drive beam while the change in energy of the witness beam is measured with a spectrometer. The accuracy and sensitivity of this measurement is limited by two main factors: (1) A time delay scanning tool is necessary and the duration of the witness beam and the jitter of the delay both act to smear out the wakefield measurement $[9,11,12]$. (2) The drive beam's self-wakefield is especially difficult to measure since the spectrometer only measures the integrated energy spectrum of the drive bunch and loses the time information so that the longitudinal electric field inside the drive beam can only be inferred. This implies that the derived quantity (for example, the transformer ratio [13-15]) must also be inferred since it depends on the peak self-wakefield experience by the drive bunch. Given these limitations there is a demand for a measurement of the wakefield experienced by the drive and witness beam in order to accurately characterize the wakefield accelerators.

In this paper, we introduce a single-shot wakefield mapping method for direct measurement of the wakefield. This technique is a modification of the traditional scanning drive-witness technique [12] wherein a long witness bunch replaces the short one and a single-shot longitudinal phase space (LPS) measurement system [16] replaces the spectrometer. This method overcomes the limitation of 
smearing out the wakefield since no scanning is needed because the long witness bunch probes the wakefield over a full wavelength and the LPS preserves the time information. Further, since the drive and witness bunches do not overlap in time then even the self-wakefield of the drive bunch can be measured. Although this paper only describes the measurement of the longitudinal wakefield, in principle, this method can also be used for measurement of the transverse wakefield.

The paper is organized as follows: in Sec. II we introduce the theory of the single-shot wakefield mapping method, which includes the introduction of long witness beam and LPS system along with the beam dynamics and resolution analysis; in Sec. III we present a simulation and an experimental demonstration of the method for the recent high transformer ratio enhanced experiment conducted at the Argonne Wakefield Accelerator (AWA) facility.

We chose this experiment as an example since it is inline with the AWA research program where two different advanced acceleration methods are studied. One approach, known as two-beam acceleration, radio frequency (rf) power is extracted from a drive decelerating structure with a waveguide and is injected into a second structure to accelerate a witness beam [17-19]. In the other approach, collinear wakefield acceleration (CWA), a leading highcharge drive bunch passes through a slow-wave structure and excites a wakefield which is used to accelerate a trailing low-charge witness beam $[6,8]$. A key difference between the two is that CWA can easily be used at high frequency since it does not rely on waveguide components which are not readily available at high frequency (e.g. THz domain). Two important figures of merit of CWA are the acceleration gradient $\left(E_{z}\right)$ and the transformer ratio (TR). For a given structure geometry and bunch shape, $E_{z}$ is proportional to the drive bunch charge and the TR is defined as the ratio of maximum accelerating field behind the drive bunch to the maximum decelerating field within the drive bunch [8]. In order to demonstrate the feasibility of the CWA scheme, it is essential to compare its design figures of merit, $E z$ and TR, with the values achieved during the experiment. The measurement of TR has not been easy with the traditional method due to the second drawback mentioned before, thus making this THz-scale CWA demonstration experiment an excellent test of our new method.

\section{SINGLE-SHOT WAKEFIELD MAPPING METHOD}

The main idea of the drive-witness method is to directly measure the wakefield by using a drive bunch to excite the wakefield and a trailing witness bunch to probe the wakefield. The key modifications of our new method are to use a long witness bunch and single-shot LPS system to achieve a more accurate wakefield mapping. We first introduce the interaction between the long witness bunch and the wakefield and then discuss the single-shot LPS system and its underlying beam dynamics.

\section{A. Long witness bunch and its interaction with wakefield}

The interaction between the wakefield and the long witness bunch can be described as follows. The situation for the drive beam is the same as with the traditional method. With no loss of generality, let us consider a drive bunch with a Gaussian longitudinal distribution (Fig. 1, solid blue line) used to excite a monopole wakefield (red dashed line) in a slow-wave structure. The electrons inside the drive bunch (navy blue dots) experience a deceleration wakefield and lose energy according to their time delay. In the energy space, the energy of the particles will change from their initial positions (in this case 0 ) to the positions of sky blue dots as indicated by arrows. The first step to improve the measurement is to use a witness bunch with a long temporal duration, but with low charge, as the new probe. Here "long" means that the length of the witness bunch can be compared to the wavelength of the wakefield being measured, as shown in Fig. 1 where red dots occupy one wavelength in the time domain. The energy change of electrons inside the witness bunch follows the wakefield amplitude versus time. Therefore, the sky blue dots and the light red dots, i.e. the energy of the drive and (long) witness bunch, contain a map of the wakefield and if we can measure the energy gain of the drive and witness at each time slice then we will have measured the longitudinal wakefield in a single shot. This long witness technique does not need a scanning tool which allows us to overcome the first drawback mentioned in the introduction part.

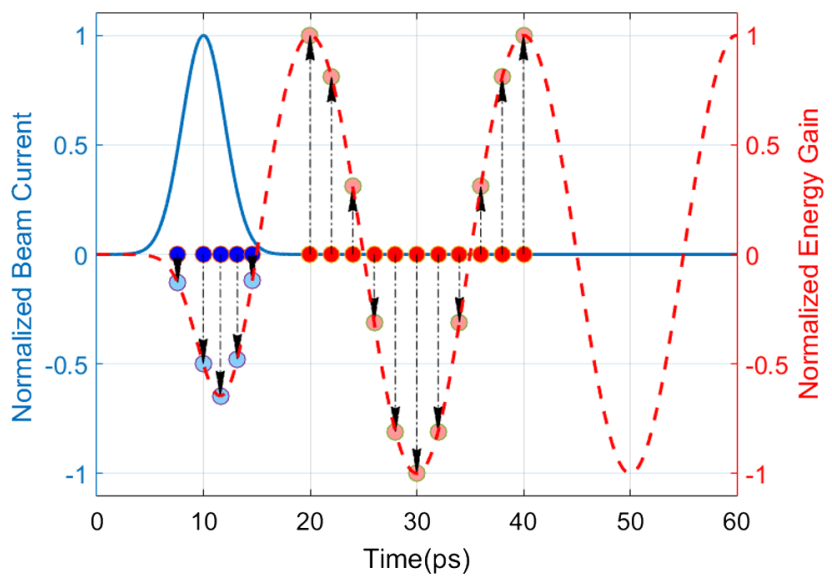

FIG. 1. Illustration of the interaction between drive and long witness. The solid blue line indicates the bunch current profile, the dashed red line is its wakefield. The navy blue/sky blue dots represent the electrons inside the drive bunch with/without wakefield, the cardinal/light red dots are the electron inside a long witness bunch with/without wakefield. 
Now we can go to the second step in improving the measurement which could overcome the second drawback of the traditional method by replacing the spectrometer with a single-shot LPS measurement system based on a transverse deflecting cavity and a spectrometer. This system is described in detail in the following text.

\section{B. Longitudinal phase space mapping}

The single-shot LPS measurement system consists of a transverse deflecting cavity (TDC), a spectrometer and focusing optics. The spectrometer bends the beam in the horizontal direction and the TDC kicks it in the vertical direction so that the energy and the temporal distribution are projected onto two orthogonal axes in a twodimensional surface. In the following text, the $x$ represents the horizontal direction and the $y$ refers to vertical direction. The single-shot LPS beam line layout we used for the experiment is shown in Fig. 2.

The two quadrupoles located between the TDC and spectrometer are used to focus the beam in the $x$ and $y$ directions to improve the resolution.

In this section, we present the beam dynamics analysis to illustrate how this system will work for wakefield mapping and an example of resolution analysis for our experimental setup in the AWA facility.

\section{Thin-lens first order beam dynamics}

We first analyze the LPS system using thin-lens approximations for the TDC and quadrupoles. This simplification is useful to understand the beam dynamics while also giving reasonably accurate results. We use coordinates $\left(x, x^{\prime}, y, y^{\prime}, z, \delta\right)$ to represent the six-dimension phase space of a particle, where $x, y, z$ refer to the particle coordinates with respect to bunch barycenter, $x^{\prime} \equiv p_{x} / p_{z}, y^{\prime} \equiv p_{y} / p_{z}$, $\delta \equiv\left(p-p_{0}\right) / p_{0}$ stand for transverse divergence and relative energy spread. All of the following analysis is under the relativistic approximation (reciprocal Lorentz factor $1 / \gamma \rightarrow 0$ and $\beta \rightarrow 1)$.

The first order beam transport matrix from Position A to Position B in Fig. 2 can be expressed as

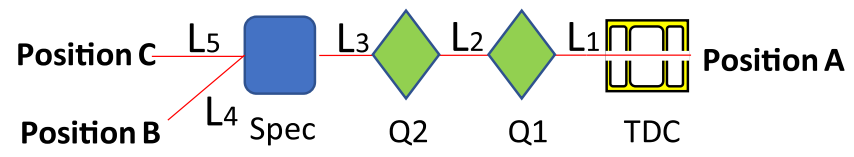

FIG. 2. LPS measurement system layout, Spec, Q, TDC, L stand for spectrometer, quadrupole, transverse deflecting cavity and drift with length L. Position A represents the location at the beginning, $\mathrm{B}$ and $\mathrm{C}$ refer to beam observation stations at the bending and the straight section after the spectrometer, separately.

$$
\begin{aligned}
M_{A B}= & M_{D}\left(L_{4}\right) \times M_{S f}(\theta) \times M_{S}(\theta) \times M_{S f}(0) \\
& \times M_{D}\left(L_{3}\right) \times M_{Q}\left(f_{2}\right) \times M_{D}\left(L_{2}\right) \\
& \times M_{Q}\left(f_{1}\right) \times M_{D}\left(L_{1}\right) \times M_{T}(0) \\
= & {\left[\begin{array}{cccccc}
R_{11_{B}} & R_{12_{B}} & R_{13_{B}} & 0 & 0 & R_{16_{B}} \\
R_{21_{B}} & R_{22_{B}} & R_{23_{B}} & 0 & 0 & R_{26_{B}} \\
0 & 0 & R_{33_{B}} & R_{34_{B}} & R_{35_{B}} & 0 \\
0 & 0 & R_{43_{B}} & R_{44_{B}} & R_{45_{B}} & 0 \\
R_{51_{B}} & R_{52_{B}} & R_{53_{B}} & 0 & 1 & R_{56_{B}} \\
0 & 0 & \kappa & 0 & 0 & 1
\end{array}\right], }
\end{aligned}
$$

where $M_{D}, M_{S f}, M_{S}, M_{Q}$ and $M_{T}$ denote the transport matrices of drift, edge focusing effect of spectrometer, spectrometer, quadrupole and TDC; their explicit expressions can be found in Appendix A. Each element $R_{i j_{B}}$ is simplified and shown in Appendix B.

Considering Eq. (1), beam horizontal and vertical coordinates at Position B can be expressed as

$$
\begin{aligned}
& x_{B}=R_{11_{B}} x_{A}+R_{12_{B}} x_{A}^{\prime}+R_{13_{B}} y_{A}+R_{16_{B}} \delta_{A} \\
& y_{B}=R_{33_{B}} y_{A}+R_{34_{B}} y_{A}^{\prime}+R_{35_{B}} z_{A} .
\end{aligned}
$$

If we minimize the contributions from the initial horizontal and vertical terms in Eq. (2), then the energy and temporal terms will dominate in $x_{B}$ and $y_{B}$. In other words, the longitudinal phase space can be taken from the $x-y$ cross section of the beam image using a beam observation tool, for instant, a fluorescent screen. Note that there is an $R_{13_{B}}$ term which means final $x_{B}$ is correlated with initial $y_{A}$, since this vertical term and other vertical terms contributing to final $y_{B}$ cannot be minimized simultaneously, a vertical slit should be located in front of TDC to mitigate the affection from $y_{A}$ on $x_{B}$. The horizontal beam size and vertical beam size at Position $B$ can be written by

$$
\begin{aligned}
\sigma_{x_{B}}^{2}= & R_{11_{B}}^{2} \sigma_{x_{A}}^{2}+R_{12_{B}}^{2} \sigma_{x_{A}^{\prime}}^{2}+R_{13_{B}}^{2} \sigma_{y_{A}}^{2}+R_{16_{B}}^{2} \sigma_{\delta_{A}}^{2} \\
& +2 R_{11_{B}} R_{12_{B}} \sigma_{x_{A} x_{A}^{\prime}}+2 R_{11_{B}} R_{13_{B}} \sigma_{x_{A} y_{A}} \\
& +2 R_{11_{B}} R_{16_{B}} \sigma_{x_{A} \delta_{A}}+2 R_{12_{B}} R_{13_{B}} \sigma_{x_{A}^{\prime} y_{A}} \\
& +2 R_{12_{B}} R_{16_{B}} \sigma_{x_{A}^{\prime} \delta_{A}}+2 R_{13_{B}} R_{16_{B}} \sigma_{y_{A} \delta_{A}} \\
\sigma_{y_{B}}^{2}= & R_{33_{B}}^{2} \sigma_{y_{A}}^{2}+R_{34_{B}}^{2} \sigma_{y_{A}^{\prime}}^{2}+R_{35_{B}} \sigma_{z_{A}}^{2}+2 R_{33_{B}} R_{34_{B}} \sigma_{y_{A} y_{A}^{\prime}} \\
& +2 R_{33_{B}} R_{35_{B}} \sigma_{y_{A} z_{A}}+2 R_{34_{B}} R_{35_{B}} \sigma_{y_{A}^{\prime} z_{A}} .
\end{aligned}
$$

Two quadrupole magnets in the measurement beam line can be used to minimize the contribution of initial horizontal and vertical terms in Eq. (3). However, optimization of these quadrupoles during the experiment is impractical, due to the difficulty of finding the analytical solution to implement during the experiment. Here we introduce an experimental method to find the optimal quadrupoles setting to minimize the initial transverse effects. An additional 
screen is introduced at the location $\mathrm{C}$ where the contributions to beam size from the initial transverse are linear to the one at location B. In this way the quadrupoles setting can be found by watching the beam size at location $\mathrm{C}$ during the operation. Its principle can be stated as follows: first, consider the transport matrix from A to $\mathrm{C}$ when the TDC and spectrometer are off,

$$
\begin{aligned}
M_{A C}= & M_{D}\left(L_{5}\right) \times M_{D}(b) \times M_{D}\left(L_{3}\right) \times M_{Q}\left(f_{2}\right) \\
& \times M_{D}\left(L_{2}\right) \times M_{Q}\left(f_{1}\right) \times M_{D}\left(L_{1}\right) \\
= & {\left[\begin{array}{cccccc}
R_{11_{C}} & R_{12_{C}} & 0 & 0 & 0 & 0 \\
R_{22_{C}} & R_{22_{C}} & 0 & 0 & 0 & 0 \\
0 & 0 & R_{33_{C}} & R_{34_{C}} & 0 & 0 \\
0 & 0 & R_{43_{C}} & R_{44_{C}} & 0 & 0 \\
0 & 0 & 0 & 0 & 1 & 0 \\
0 & 0 & 0 & 0 & 0 & 1
\end{array}\right], }
\end{aligned}
$$

where $b \equiv \rho \sin \theta$ is the dipole effective length, $\theta$ refers to bending angle, $\rho$ represents the radius of curvature of bending trajectory. All the expressions of $R_{i j_{C}}$ can be found in Appendix B. Similar to Eq. (3), the horizontal and vertical beam size at Position $\mathrm{C}$ can be expressed as

$$
\begin{aligned}
& \sigma_{x_{C}}^{2}=R_{11_{C}}^{2} \sigma_{x_{A}}^{2}+R_{12_{C}}^{2} \sigma_{x_{A}^{\prime}}^{2}+2 R_{11_{C}} R_{12_{C}} \sigma_{x_{A} x_{A}^{\prime}} \\
& \sigma_{y_{C}}^{2}=R_{33_{C}}^{2} \sigma_{y_{A}}^{2}+R_{34_{C}}^{2} \sigma_{y_{A}^{\prime}}^{2}+2 R_{33_{C}} R_{34_{C}} \sigma_{y_{A} y_{A}^{\prime}} .
\end{aligned}
$$

We noticed that when the drift length $L_{4}$ and $L_{5}$ have the following relationship,

$$
\begin{aligned}
L_{5}= & \frac{L_{4}}{\cos ^{2} \theta}+\rho \tan \theta-\rho \sin \theta \\
L_{5}= & \frac{\rho\left[\theta \rho+L_{4}\left(1-\theta \tan \phi_{e}\right)\right]}{\rho\left(1-\theta \tan \phi_{i}\right)-L_{4}\left[\tan \phi_{i}+\tan \phi_{e}\left(1-\theta \tan \phi_{i}\right)\right]} \\
& -\rho \sin \theta,
\end{aligned}
$$

where $\tan \phi_{i}$ and $\tan \phi_{e}$ refer to the effective vertical focusing angle at incident and exit pole of the dipole due to edge focusing effect (their explicit expressions are shown in Appendix A), then the horizontal and vertical related elements in matrix $M_{A B}$ and $M_{A C}$ have the dependency as

$$
\begin{array}{ll}
R_{11_{B}}=\zeta_{x} R_{11_{C}} ; & R_{12_{B}}=\zeta_{x} R_{12_{C}} \\
R_{33_{B}}=\zeta_{y} R_{33_{C}} ; & R_{34_{B}}=\zeta_{y} R_{34_{C}},
\end{array}
$$

where $\zeta_{x}=\cos \theta$

$\zeta_{y}=\frac{\rho\left(1-\theta \tan \phi_{i}\right)-L_{4}\left[\tan \phi_{i}+\tan \phi_{e}\left(1-\theta \tan \phi_{i}\right)\right]}{\rho}$.

Note here $\zeta_{x}$ and $\zeta_{y}$ only depend on the spectrometer geometry and the drift length behind the spectrometer. Substituting Eqs. (5) and (7) into Eq. (3), we get the beam horizontal and vertical size at Position B as

$$
\begin{aligned}
\sigma_{x_{B}}^{2}= & \zeta_{x}^{2} \sigma_{x_{C}}^{2}+R_{16_{B}}^{2} \sigma_{\delta_{A}}^{2}+R_{13_{B}}^{2} \sigma_{y_{A}}^{2}+2 R_{11_{B}} R_{16_{B}} \sigma_{x_{A} \delta_{A}} \\
& +2 R_{12_{B}} R_{16_{B}} \sigma_{x_{A}^{\prime} \delta_{A}}+2 R_{11_{B}} R_{13_{B}} \sigma_{x_{A} y_{A}} \\
& +2 R_{12_{B}} R_{13_{B}} \sigma_{x_{A}^{\prime} y_{A}}+2 R_{13_{B}} R_{16_{B}} \sigma_{y_{A} \delta_{A}} \\
\sigma_{y_{B}}^{2}= & \zeta_{y}^{2} \sigma_{y_{C}}^{2}+R_{35_{B}}^{2} \sigma_{z_{A}}^{2}+2 R_{33_{B}} R_{35_{B}} \sigma_{y_{A} z_{A}} \\
& +2 R_{34_{B}} R_{35_{B}} \sigma_{y_{A}^{\prime} z_{A}} .
\end{aligned}
$$

We now describe how to use the quads to minimize the effect of the transverse phase space (TPS) on the resolution of the LPS measurement. The first terms in the above expressions for the horizontal (or vertical) beam size at position $\mathrm{B}$ are proportional to the spot size at position $\mathrm{C}$. Therefore, if quads setting are found to focus the beam to a smallest beam size at Position C (with TDC and spectrometer off), it will minimize the effect of the initial horizontal and vertical terms on the LPS measurement. This provides a simple practical method that can be used during the experiment to suppress the error of LPS measurement caused by beam initial TPS. For example, in an experiment following steps can help us to achieve better measurement for LPS. First, design the bending and straight section drift length $\left(L_{4}\right.$ and $\left.L_{5}\right)$ to be matched with Eq. (6). Second, tune the doublets strength to make as small beam size as possible at Position $\mathrm{C}$ with TDC and spectrometer off, then turn on the TDC and spectrometer to analyze the beam image at Position B.

Inspecting Eq. (6), when the dipole geometric length $\mathrm{b}$ is determined, the $L_{4}$ and $L_{5}$ are only dependent with bending angle $\theta$, i.e. for a specific $\theta$ value, there is only one solution for Eq. (6). In Fig. 3, we present an example of $L_{4}$ and $L_{5}$ versus different bending angle $\theta$ when $b=0.365 \mathrm{~m}$.

\section{Thick-cavity effect}

In our case, the thick-lens effect of the quadrupole will not break the zero terms in Eqs. (1) and (4), which means the Eq. (9) will not add any additional terms related to beam phase space compared to the thin-lens quadrupole case. The only affection is that the explicit expressions of $R_{11_{B}}, R_{12_{B}}$, $R_{33_{B}}$ and $R_{34_{B}}$ in Eq. (9) are different, which would not affect our following analysis. Therefore, the thin-lens approximation for the quadrupole is good enough so we keep it. However, the thick-cavity matrix of TDC could introduce new terms into Eq. (9) so that in this section we take the TDC finite length of $L_{T}$ into consideration. 
The thick-cavity transport matrix of a TDC is shown in Eq. (A2); we could rewrite the transport matrix from A to B $\left(M_{A B}\right)$ according to Eq. (1) with the TDC thick-cavity form,

$$
M_{A B}=\left[\begin{array}{cccccc}
R_{11_{B}} & R_{12_{B}}^{*} & R_{13_{B}} & R_{14_{B}}^{*} & R_{15_{B}}^{*} & R_{16_{B}} \\
R_{21_{B}} & R_{22_{B}}^{*} & R_{23_{B}} & R_{24_{B}}^{*} & R_{25_{B}}^{*} & R_{26_{B}} \\
0 & 0 & R_{33_{B}} & R_{34_{B}}^{*} & R_{35_{B}}^{*} & 0 \\
0 & 0 & R_{43_{B}} & R_{44_{B}}^{*} & R_{45_{B}}^{*} & 0 \\
R_{51_{B}} & R_{52_{B}}^{*} & R_{53_{B}} & R_{54_{B}}^{*} & R_{55_{B}}^{*} & R_{56_{B}} \\
0 & 0 & \kappa & \frac{\kappa L_{T}}{2} & \frac{\kappa^{2} L_{T}}{4} & 1
\end{array}\right] .
$$

The superscript $*$ means the new elements which are different from the ones of the thin-lens matrix; all the explicit expressions of matrix elements are exhibited in
Appendix B. In the same way, we could also derive the thick-cavity form of transport matrix $M_{A C}$ as

$$
M_{A C}=\left[\begin{array}{cccccc}
R_{11_{C}} & R_{12_{C}}^{*} & 0 & 0 & 0 & 0 \\
R_{21_{C}} & R_{22_{C}}^{*} & 0 & 0 & 0 & 0 \\
0 & 0 & R_{33_{C}} & R_{34_{C}}^{*} & 0 & 0 \\
0 & 0 & R_{43_{C}} & R_{44_{C}}^{*} & 0 & 0 \\
0 & 0 & 0 & 0 & 1 & 0 \\
0 & 0 & 0 & 0 & 0 & 1
\end{array}\right] .
$$

The method to minimize the effect of the initial TPS still works for the thick-cavity case, which means Eq. (9) can be updated to

$$
\begin{aligned}
\sigma_{x_{B}}^{2}= & \zeta_{x}^{2} \sigma_{x_{C}}^{2}+R_{13_{B}}^{2} \sigma_{y_{A}}^{2}+R_{14_{B}}^{* 2} \sigma_{y_{A}^{\prime}}^{2}+R_{15_{B}}^{* 2} \sigma_{z_{A}}^{2}+R_{16_{B}}^{2} \sigma_{\delta_{A}}^{2}+2 R_{11_{B}} R_{16_{B}} \sigma_{x_{A} \delta_{A}}+2 R_{12_{B}}^{*} R_{16_{B}} \sigma_{x_{A}^{\prime} \delta_{A}}+2 R_{13_{B}} R_{16_{B}} \sigma_{y_{A} \delta_{A}} \\
& +2 R_{14_{B}}^{*} R_{16_{B}} \sigma_{y_{A}^{\prime} \delta_{A}}+2 R_{15_{B}}^{*} R_{16_{B}} \sigma_{z_{A} \delta_{A}}+2 R_{11_{B}} R_{15_{B}}^{*} \sigma_{x_{A} z_{A}}+2 R_{12_{B}}^{*} R_{15_{B}}^{*} \sigma_{x_{A}^{\prime} z_{A}}+2 R_{13_{B}} R_{15_{B}}^{*} \sigma_{y_{A} z_{A}}+2 R_{14_{B}}^{*} R_{15_{B}}^{*} \sigma_{y_{A}^{\prime} z_{A}} \\
& +2 R_{11_{B}} R_{14_{B}}^{*} \sigma_{x_{A} y_{A}^{\prime}}+2 R_{12_{B}}^{*} R_{14_{B}}^{*} \sigma_{x_{A}^{\prime} y_{A}^{\prime}}+2 R_{13_{B}} R_{14_{B}}^{*} \sigma_{y_{A} y_{A}^{\prime}}+2 R_{11_{B}} R_{13_{B}} \sigma_{x_{A} y_{A}}+2 R_{12_{B}}^{*} R_{13_{B}} \sigma_{x_{A}^{\prime} y_{A}} \\
\sigma_{y_{B}}^{2}= & \zeta_{y}^{2} \sigma_{y_{C}}^{2}+R_{35_{B}}^{* 2} \sigma_{z_{A}}^{2}+2 R_{33_{B}} R_{35_{B}}^{*} \sigma_{y_{A} z_{A}}+2 R_{34_{B}}^{*} R_{35_{B}}^{*} \sigma_{y_{A}^{\prime} z_{A}} .
\end{aligned}
$$

From the equation above we can see that the energy projection is deteriorated by multiple terms such as nonzero $R_{14_{B}}^{*}$ and $R_{15_{B}}^{*}$ which are proportional to the TDC strength $\kappa$ according to Appendix A; it means that though the higher TDC strength could increase temporal measurement resolution, it could also deteriorate the resolution of energy measurement due to thick-cavity effect.

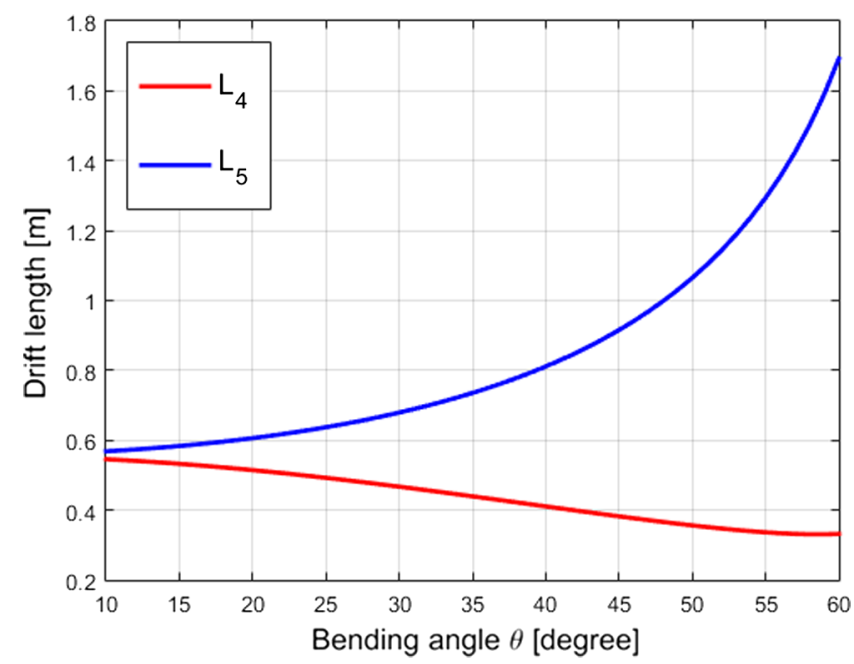

FIG. 3. Drift length $L_{4}$ and $L_{5}$ at different bending angle when spectrometer geometer length $\mathrm{b}$ is $0.365 \mathrm{~m}$.

\section{Resolution analysis}

The definition of resolution is the minimum difference of the signal that can be distinguished. In Eq. (12), in order to study the energy information in $\sigma_{x_{B}}$ and time information in $\sigma_{y_{B}}$, we can define the energy and temporal resolution as

$$
\sigma_{\delta}=\frac{\sigma_{x_{\text {other }}}}{R_{16_{B}}^{*}} \quad \sigma_{t}=\frac{\sigma_{y_{\text {other }}}}{v_{c} R_{35_{B}}^{*}}
$$

where $v_{c}$ is the speed of light, $\sigma_{x_{\text {other }}}$ and $\sigma_{y_{\text {other }}}$ are the terms contributed by other terms except energy or time. According to Eq. (12), their explicit expressions can be expressed as

$$
\begin{aligned}
\sigma_{x_{\text {other }}}^{2}= & \zeta_{x}^{2} \sigma_{x_{C}}^{2}+R_{13_{B}}^{2} \sigma_{y_{A}}^{2}+R_{14_{B}}^{* 2} \sigma_{y_{A}^{\prime}}^{2}+R_{15_{B}}^{* 2} \sigma_{z_{A}}^{2} \\
& +2 R_{11_{B}} R_{15_{B}}^{*} \sigma_{x_{A} z_{A}}+2 R_{12_{B}}^{*} R_{15_{B}}^{*} \sigma_{x_{A}^{\prime} z_{A}} \\
& +2 R_{13_{B}} R_{15_{B}}^{*} \sigma_{y_{A} z_{A}}+2 R_{14_{B}}^{*} R_{15_{B}}^{*} \sigma_{y_{A}^{\prime} z_{A}} \\
& +2 R_{11_{B}} R_{14_{B}}^{*} \sigma_{x_{A} y_{A}^{\prime}}+2 R_{12_{B}}^{*} R_{14_{B}}^{*} \sigma_{x_{A}^{\prime} y_{A}^{\prime}} \\
& +2 R_{13_{B}} R_{14_{B}}^{*} \sigma_{y_{A} y_{A}^{\prime}}+2 R_{11_{B}} R_{13_{B}} \sigma_{x_{A} y_{A}}+2 R_{12_{B}}^{*} R_{13_{B}} \sigma_{x_{A}^{\prime} y_{A}} \\
\sigma_{y_{\text {other }}}^{2}= & \zeta_{y}^{2} \sigma_{y_{C}}^{2} .
\end{aligned}
$$

Theoretically, we could calculate the resolution through Eqs. (13) and (14); it is affected by many variables such as spectrometer bending angle $\theta$ and TDC kick strength $\kappa$. Since the $\theta$ is not easy to change once the chamber is 
TABLE I. Beam phase space Courant-Snyder (CS) parameters of numerical example at Position A.

\begin{tabular}{lrc}
\hline \hline Parameters & Value & Unit \\
\hline$\alpha_{x}$ & 1.1 & \\
$\beta_{x}$ & 49.9 & $\mathrm{~m}$ \\
$\epsilon_{x}$ & 26.4 & $\mu \mathrm{m}$ \\
$\alpha_{y}$ & 1.2 & \\
$\beta_{y}$ & 50.9 & $\mathrm{~m}$ \\
$\epsilon_{y}$ & 26.0 & $\mu \mathrm{m}$ \\
$\alpha_{z}$ & -3.6 & \\
$\beta_{z}$ & 1.2 & $\mathrm{~m}$ \\
$\epsilon_{z}$ & 140.2 & $\mu \mathrm{m}$ \\
$\gamma$ & 95.16 & \\
\hline \hline
\end{tabular}

fabricated and the $\kappa$ is limited by the rf power, both of them should be well designed prior to other variables such as drift length. Thus we present the analysis of resolution versus bending angle $\theta$ and TDC kick strength $\kappa$ to give a guide when designing the LPS measurement system.

The resolution at different bending angles and kick strengths is calculated in the following steps. First, according to the geometry of our existing hardwares, we set the drift length $L_{1}=0.6 \mathrm{~m}, L_{2}=0.5 \mathrm{~m}, L_{3}=0.5 \mathrm{~m}$, TDC length $L_{T}=0.23 \mathrm{~m}$ and spectrometer length $b=0.365 \mathrm{~m}$. The $L_{4}$ and $L_{5}$ are set to the values complying to Eq. (6) for each different $\theta$ value. At this point, the geometric variables of the LPS measurement system are settled. Second, we choose particle tracking simulation since it is not easy to
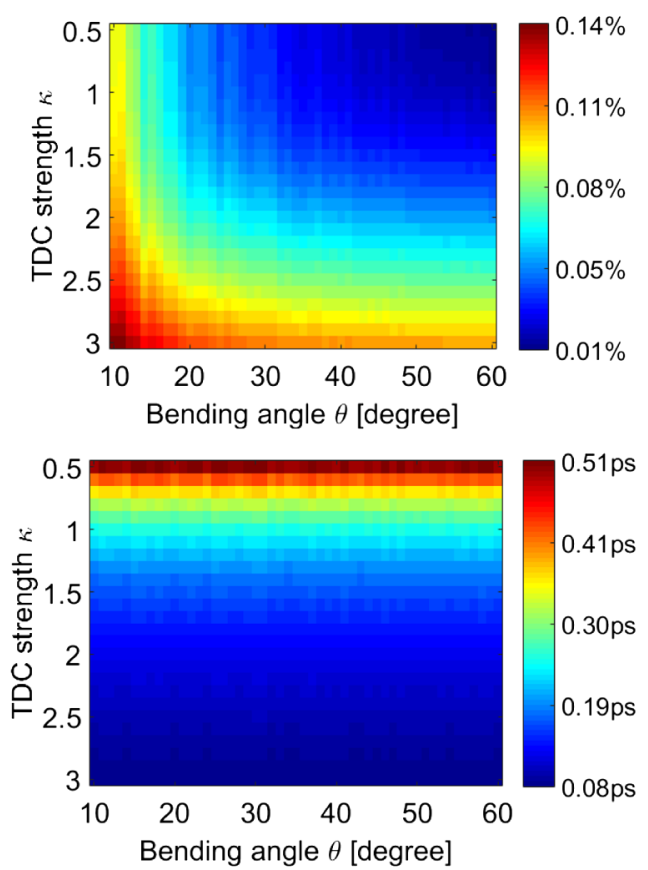

FIG. 4. Energy (top figure) and temporal (bottom figure) measurement resolution versus bending angle $\theta$ and TDC strength $\kappa$. include the function of slit in matrix calculation. As an example, we used a typical 6D beam phase space CS parameters, which is obtained from the simulation based on the AWA beam line model when electron bunch charge is $15 \mathrm{nC}$, see Table I. As stated before, a slit with $100-\mu \mathrm{m}$ vertical aperture is placed before TDC to eliminate the initial vertical terms affection on energy measurement. The doublets quadrupole settings are numerically found to achieve the minimum $\mathrm{x}$ and $\mathrm{y}$ beam size at Position $\mathrm{C}$ for each $\theta$ and $\kappa$ when TDC and spectrometer are turned off. Finally, the beam phase space at Position B is achieved in the particle tracking simulation, $\sigma_{x_{\text {other }}}$ and $\sigma_{y_{\text {other }}}$ are calculated according to Eq. (14), then the resolution could be obtained according to Eq. (13).

The resolution result with respect to each $(\theta, \kappa)$ point is shown in Fig. 4. Higher TDC strength could provide better temporal resolution, but it would deteriorate energy resolution mainly due to the $R_{15 B}^{* 2} \sigma_{z_{A}}^{2}$ term in Eq. (14). On the other hand, the dispersion of dipole will increase as the bending angle increases which results in a better energy resolution.

\section{DEMONSTRATION OF MAPPING METHOD}

In order to demonstrate the feasibility of the mapping method, we present the start-to-end simulation and the experiment results based on the high transformer ratio experiment [20] conducted at the AWA beam line. The AWA beam line is shown in Fig. 5; it can be divided into four sections. The first section consists of a L-band 1.5-cell photoinjector and six standing-wave linacs acting as the acceleration elements. In the second section, the beam is sent to the emittance exchange (EEX) beam line which has the function of exchanging the beam longitudinal space with transverse space $[21,22]$. The third section is the wakefield generation section which consists of a dielectriclined slab (DLS) as the wakefield accelerating structure. It consists of a $150-\mu \mathrm{m}$ thickness layer of quartz with permittivity $\epsilon_{r}=3.75$ and copper coating on one side. Its width in the $\mathrm{x}$ direction is $1.27 \mathrm{~cm}$ and the gap of vacuum in the y direction ranges from 0 to $3.1 \mathrm{~cm}$ corresponding to different frequencies of eigenmode. The last section is the LPS measurement system, which has the same configuration as shown in Fig. 2. Its geometric parameters are exhibited in Table II. The spectrometer bending angle $\theta$ and TDC kick strength $\kappa$ are designed to be $20^{\circ}$ and 2 which are determined by our hardwares in the AWA. According to the resolution result in Fig. 4, the energy resolution here is $0.06 \%$ and the time resolution is 0.124 ps corresponding to frequency up to $8 \mathrm{THz}$.

\section{A. Start-to-end simulation demonstration}

The start-to-end simulation is implemented by General Particle Tracer (GPT) code, which includes the space charge effect and coherent synchrotron radiation 


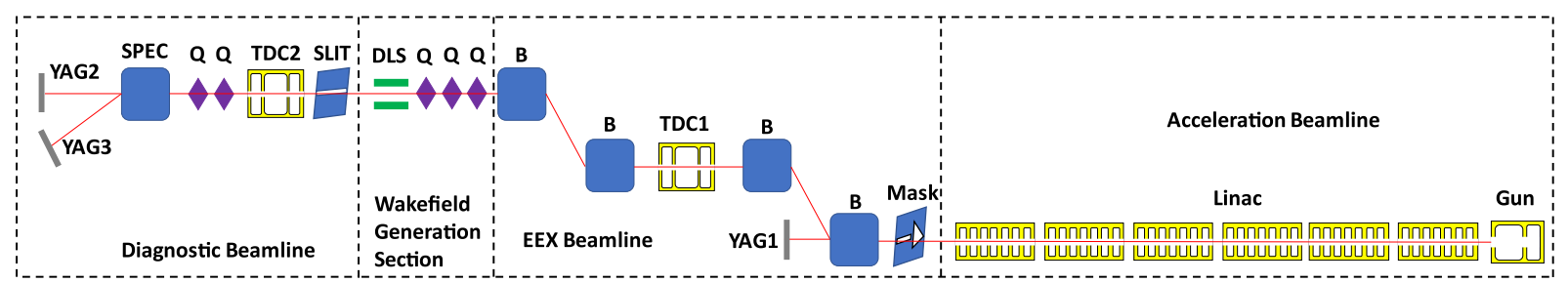

FIG. 5. AWA beam line schematic. B, Q, TDC, DLS, SPEC stand for bending dipole, quadrupole, transverse deflecting cavity, dielectric-lined slab structure and spectrometer, respectively. YAG is the screen for beam observation.

effect $[23,24]$. An $8-\mathrm{MeV}$ bunch is generated by the photoinjector and accelerated to energy of $48 \mathrm{MeV}$ by linacs. A transverse mask is placed at the entrance of the EEX beam line to intercept the beam to a triangular (drive) and a rectangular (witness) shape transversely as shown in Fig. 6. After the phase-space exchanging process, this feature is mapped into longitudinal at the EEX exit so that a triangular drive bunch and a long witness bunch are generated for wakefield measurement.

The drive bunch longitudinal profile at the EEX exit and its predicted wakefield generated in DLS are shown in Fig. 7. The tail of the triangle is not as sharp as in the initial transverse space, which is due to the perturbation of coherent synchrotron radiation [21,25]. The total length of the witness bunch is designed to be longer than one wavelength of wakefield fundamental mode as described in Sec. II A, thus the wakefield can be measured with single shot.

Figure 8 shows an example of single-shot LPS mapping. Part (a) is the original LPS before the TDC2 but after the slit, which corresponds to position A in Fig. 2. After setting the quadrupoles according to the method stated above to suppress the affection from initial transverse phase space, the beam $x-y$ cross image taken at YAG3 is shown in (b). Since the $\mathrm{x}$ represents the energy and $\mathrm{y}$ refers to time, we could reconstruct the LPS using this image and plot it with the original one at the same picture, see (c). Since we concentrate on the wakefield versus time t, we could calculate the average energy at each time slice so that the average profile of LPS is obtained and shown in (d). The average profiles from both cases match well with each

TABLE II. LPS diagnostic system geometric parameters.

\begin{tabular}{lcc}
\hline \hline Parameters & Value & Unit \\
\hline$L_{1}$ & 0.600 & $\mathrm{~m}$ \\
$L_{2}$ & 0.500 & $\mathrm{~m}$ \\
$L_{3}$ & 0.500 & $\mathrm{~m}$ \\
$L_{4}$ & 0.514 & $\mathrm{~m}$ \\
$L_{5}$ & 0.606 & $\mathrm{~m}$ \\
$L_{T}$ & 0.230 & $\mathrm{~m}$ \\
$b$ & 0.365 & $\mathrm{~m}$ \\
$\theta$ & 20.0 & degree \\
$\kappa$ & 2.00 & $\mathrm{~m}^{-1}$ \\
\hline \hline
\end{tabular}

other which indicates that the beam image at YAG3 is a good representative of LPS before TDC2.

Next, the wakefield of the drive bunch can be derived by the following procedure. First, the beam images of both drive with witness and witness alone at wakefield on/off are taken at YAG3 and shown in Fig. 9. Wakefield on means that the wake function of DLS is applied in simulation and it corresponds to the experimental situation where the DLS gap is small. Conversely, wakefield off means that the wake

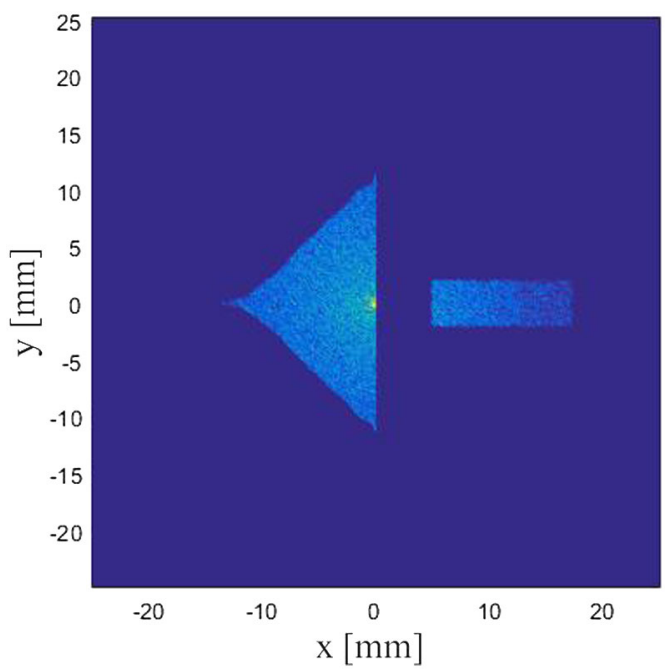

FIG. 6. Beam image right after mask at EEX entrance in the simulation. The triangle indicates the drive bunch and the long rectangle refers to witness bunch.

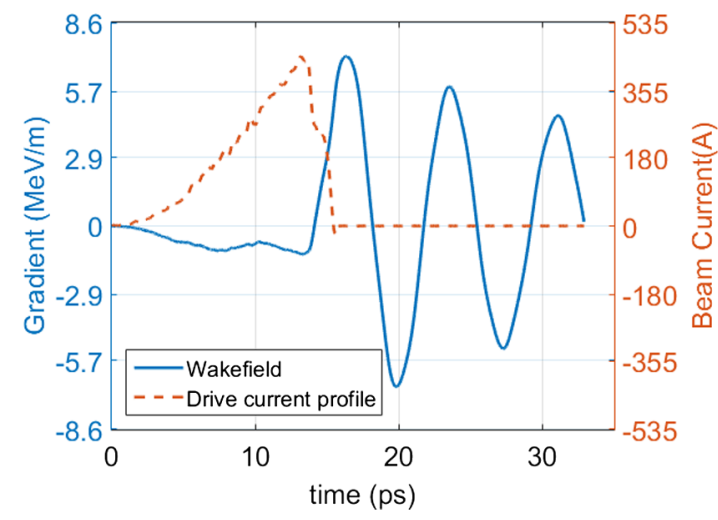

FIG. 7. Analytical wakefield of drive bunch in simulation. 

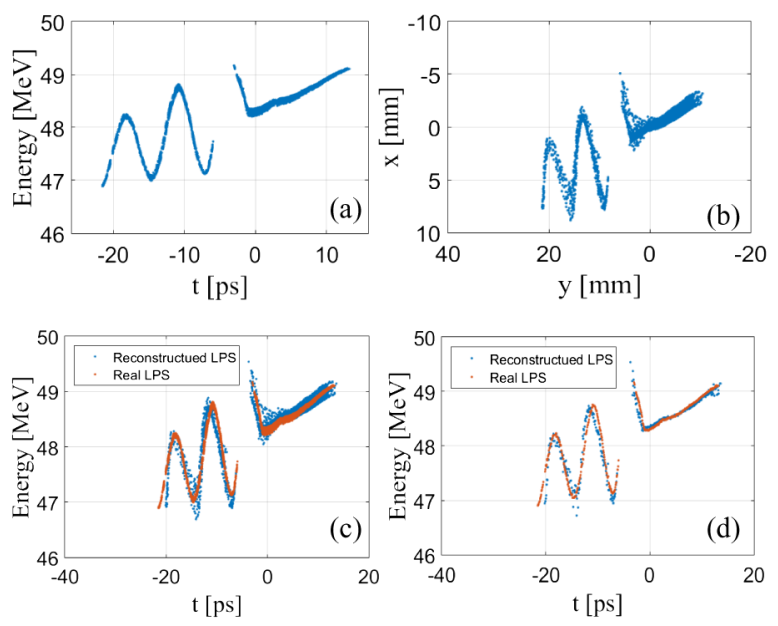

FIG. 8. Illustration of LPS mapping. (a) Original LPS before TDC2. (b) Beam image at YAG3. (c) Comparison between original and mapping LPS. (d) Comparison of average LPS profile between original and mapping LPS.

function of DLS is not applied in simulation and it corresponds to the experimental situation where the DLS gap is large. Second, calculate the average LPS profile of each image then take the difference between wakefield on and off at each time slice to achieve the measured wakefield. Note that the witness energy gain induced by itself wakefield should be subtracted out from the total amount. Then we could get the wakefield inside and behind the drive bunch from measurement image data. The measured and analytical wakefield are shown in Fig. 10. The results show that the reconstructed wakefield matches well with the analytical one which means our method is practical, thus we could use this technique to measure the wakefield in the experiment.
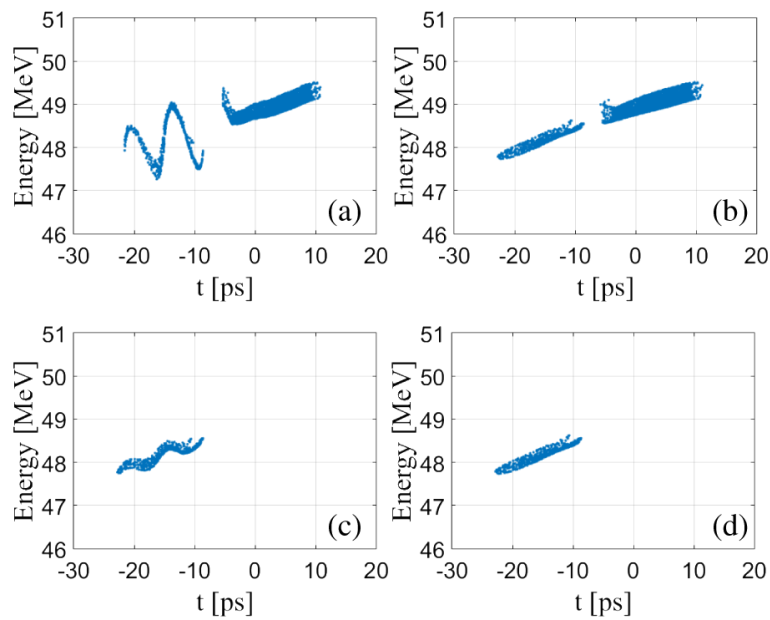

FIG. 9. Beam images at YAG3 in simulation. (a) and (b) Drive and witness when wakefield is on/off. (c) and (d) Witness when wakefield is on/off.

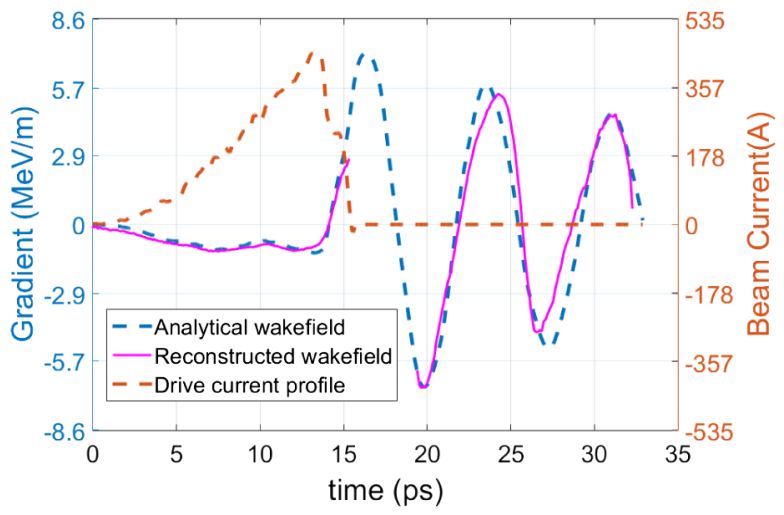

FIG. 10. Comparison between measured and analytical wakefield in simulation.

\section{B. Experiment results}

Afterwards, we conducted the experiment in the AWA beam line which is shown in Fig. 5. During the experiment, a pulsed UV laser with homogenized transverse distribution [26] is delivered into the photoinjector to generate an electron bunch with $20 \mathrm{nC}$ charge. The beam image at the EEX entrance (after the beam was shaped by the transverse mask) is shown at Fig. 11(a). At the exit of the EEX, the drive bunch temporal profile is measured by TDC2 at
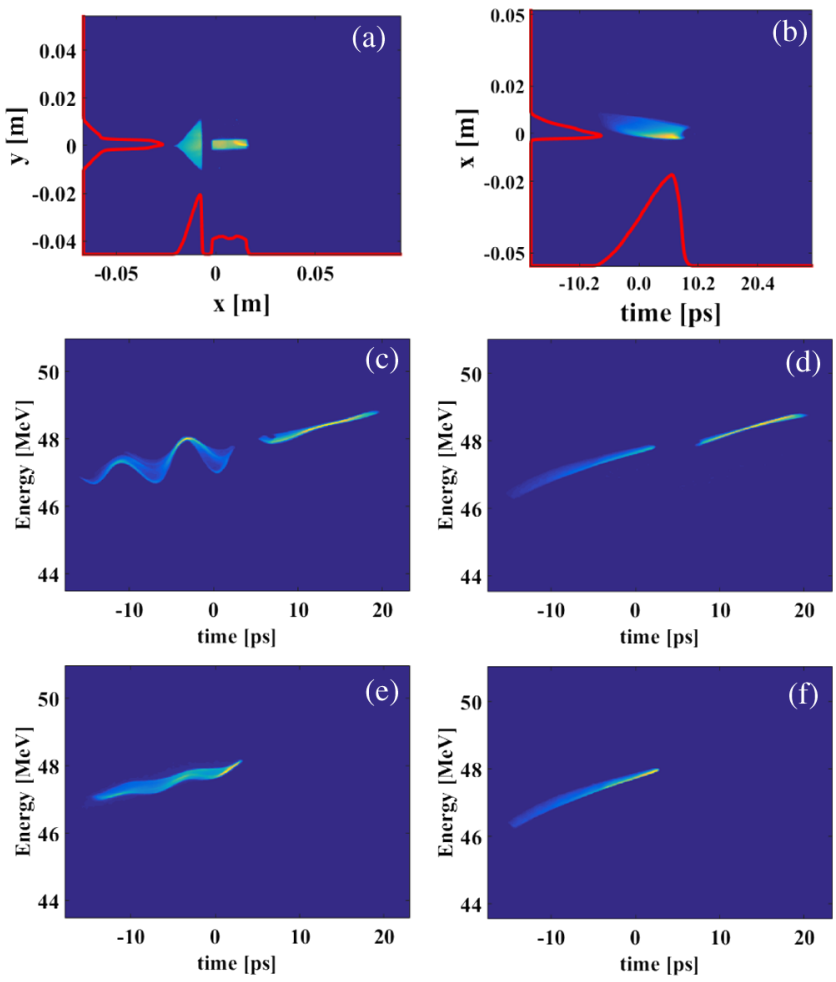

FIG. 11. Beam image from experiment: (a) Beam transverse distribution at YAG1, the red line refers to projection. (b) Longitudinal profile measurement result of drive bunch at YAG2. (c) and (d) Drive and witness bunch at YAG3 with wakefield on/ off. (e) and (f) Witness bunch at YAG3 with wakefield on/off. 


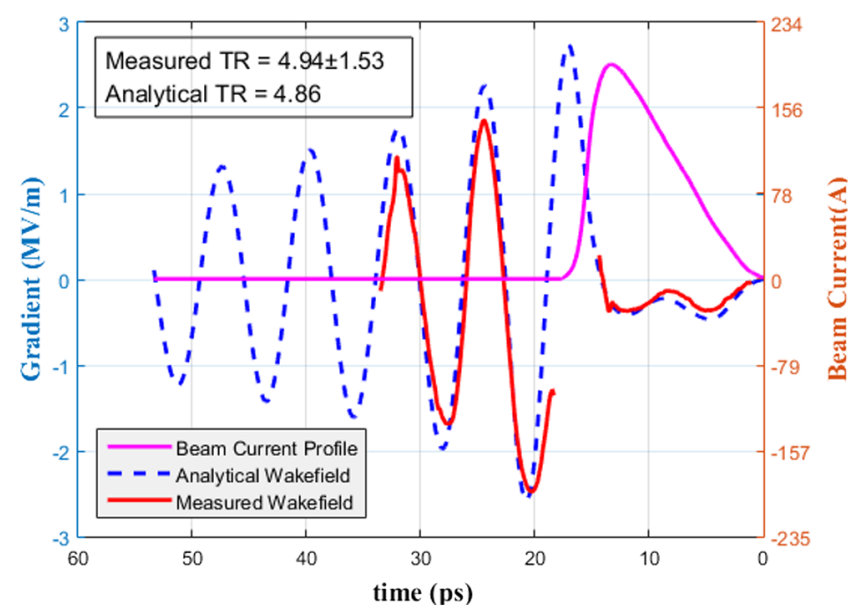

FIG. 12. Comparison between measured and analytical wakefield.

YAG2 shown in Fig. 11(b). We can see that the drive bunch has a quasitriangular distribution, although it still should have a high transformer ratio. The DLS gap size is set to be $2.1 \mathrm{~mm}$ which corresponds to the wakefield with fundamental frequency of $130.7_{-4.7}^{+5.3} \mathrm{GHz}$. The transmitted charge of drive after DLS when its gap is $2.1 \mathrm{~mm}$ is $1.66_{-0.46}^{+0.19} \mathrm{nC}$ and of witness is $0.90_{-0.07}^{+0.18} \mathrm{nC}$. Following the procedure introduced in the simulation section to construct the wakefield, the beam images at YAG3 at different cases were taken and shown in Figs. 11(c)-11(f). Here the wakefield on means the DLS gap size of $2.1 \mathrm{~mm}$ while wakefield off indicates that the two jaws of the DLS are fully open (gap size of $31 \mathrm{~mm}$ ) whose wakefield strength is a factor of 10,000 weaker than the wakefield on case.

Applying the postprocess method that was described in the simulation section above, we get the measured wakefield which is compared with the analytical wakefield and shown in Fig. 12. The analytical one is calculated using the measured longitudinal profile of the drive bunch in Fig. 11(b) and Green's function of DLS [27]. As seen in the figure, the measured wakefield inside and behind the drive bunch matches well with the analytical ones.

Here we present an application of this single-shot wakefield mapping technique. The transformer ratio is an important figure of merit in the collinear wakefield accelerator, however, due to the small loss energy of the drive bunch, it is very hard to directly measure this quantity by the traditional drive-witness method. In contrast, using this $\mathrm{THz}$ single-shot wakefield mapping technique, we can get the temporal and energy information and reconstruct the wakefield precisely so that it is possible to directly measure it with single shot. For example, from the measured wakefield shown in Fig. 12, we can calculate the ratio of maximum energy gain of the witness bunch to the maximum energy loss of the drive bunch as $4.94 \pm 1.53$, of which the error bar is computed from the average profile jitter. Here the maximum energy gain of the witness refers to the second peak after the drive bunch; it is because the drive bunch tail reaches into the first peak region so that it cannot be used for witness acceleration. The TR derived from the analytical wakefield is 4.86 , which has an excellent agreement with our measured value. It demonstrates that the wakefield measurement technique can be a general solution to solve the problem of accurate TR measurement.

\section{CONCLUSION}

In this paper, we introduced a single-shot wakefield measurement system which utilizes a long witness as the probe and single-shot longitudinal phase space mapping system. The beam dynamics of the LPS measurement system was fully discussed and expressions for its resolution were given. Furthermore, we come up with a practical approach to minimize the effect of the transverse phase space on the longitudinal phase space measurement. Both a start-to-end simulation and a proof of principle experiment performed at the AWA facility have been presented to demonstrate the feasibility of the technique. In the paper, we concentrated on the longitudinal wakefield measurement, however, this setup can be easily extended for measurement of the transverse wakefield. For example, once the drive beam is injected into the slowwave structure with an offset to the axis, it will excite the transverse wakefield and the trailing long witness bunch will receive the transverse kick. The long witness bunch phase space $p_{x}-z$ can be projected into to $\mathrm{x}-\mathrm{y}$ cross image with a TDC and a long drift. Thus the transverse wakefield $W_{\perp}(z)$ could be reconstructed. Since this method not only has the ability of measuring the long range wakefield but also of measuring the short range wakefield with high resolution, it can be a general tool for wakefield measurements.

\section{ACKNOWLEDGMENTS}

This work is supported by the U.S. Department of Energy, Offices of HEP and BES, under Contract No. DE-AC02-06CH11357. It is also funded by the National Natural Science Foundation of China (NSFC) No. 11375098. We would also like to thank Dr. S.S. Baturin who is supported by NSF Grant No. PHY-1549132 for many helpful discussions on Green's function calculation for DLS.

\section{APPENDIX A: TRANSPORT MATRIX OF BASIC ELEMENT}

A standing-wave TDC which works at $T M_{110}$ mode [28] deflecting beam in the y direction can be expressed by 


$$
M_{T}\left(L_{T}\right)=\left[\begin{array}{cccccc}
1 & L_{T} & 0 & 0 & 0 & 0 \\
0 & 1 & 0 & 0 & 0 & 0 \\
0 & 0 & 1 & L_{T} & \frac{\kappa L_{T}}{2} & 0 \\
0 & 0 & 0 & 1 & \kappa & 0 \\
0 & 0 & 0 & 0 & 1 & 0 \\
0 & 0 & \kappa & \frac{\kappa L_{T}}{2} & \frac{\kappa^{2} L_{T}}{4} & 1
\end{array}\right]
$$

where $\kappa$ represents the dimensionless kick strength, $L_{T}$ refers to the TDC length. Once the length is neglected, it degenerates to the thin-lens case as

$$
M_{T}(0)=\left[\begin{array}{cccccc}
1 & 0 & 0 & 0 & 0 & 0 \\
0 & 1 & 0 & 0 & 0 & 0 \\
0 & 0 & 1 & 0 & 0 & 0 \\
0 & 0 & 0 & 1 & \kappa & 0 \\
0 & 0 & 0 & 0 & 1 & 0 \\
0 & 0 & \kappa & 0 & 0 & 1
\end{array}\right]
$$

The transport matrix of a thin-lens quadrupole has the form of

The matrix of a dipole can be written as

$$
M_{S}(\theta)=\left[\begin{array}{cccccc}
\cos \theta & \rho \sin \theta & 0 & 0 & 0 & \rho(1-\cos \theta) \\
-\frac{\sin \theta}{\rho} & \cos \theta & 0 & 0 & 0 & \sin \theta \\
0 & 0 & 1 & \rho \theta & 0 & 0 \\
0 & 0 & 0 & 1 & 0 & 0 \\
-\sin \theta & -\rho(1-\cos \theta) & 0 & 0 & 1 & \rho(\sin \theta-\theta) \\
0 & 0 & 0 & 0 & 0 & 1
\end{array}\right]
$$

where the $\theta$ refers to bending angle, $\rho$ represents the radius of curvature of bending trajectory. In reality, the fringe field effect of a dipole must be taken into consideration since it behaves like a thin quadrupole to the beam. Its matrix can be given by

$$
M_{S f}(\varphi)=\left[\begin{array}{cccccc}
1 & 0 & 0 & 0 & 0 & 0 \\
\frac{\tan \varphi}{\rho} & 1 & 0 & 0 & 0 & 0 \\
0 & 0 & 1 & 0 & 0 & 0 \\
0 & 0 & -\frac{\tan \phi_{v}}{\rho} & 1 & 0 & 0 \\
0 & 0 & 0 & 0 & 1 & 0 \\
0 & 0 & 0 & 0 & 0 & 1
\end{array}\right]
$$

$$
M_{Q}(f)=\left[\begin{array}{cccccc}
1 & 0 & 0 & 0 & 0 & 0 \\
-\frac{1}{f} & 1 & 0 & 0 & 0 & 0 \\
0 & 0 & 1 & 0 & 0 & 0 \\
0 & 0 & \frac{1}{f} & 1 & 0 & 0 \\
0 & 0 & 0 & 0 & 1 & 0 \\
0 & 0 & 0 & 0 & 0 & 1
\end{array}\right]
$$

where $f$ refers to the focal length.

The well-known transport matrix of a drift with length $L$ is

$$
M_{D}(L)=\left[\begin{array}{llllll}
1 & L & 0 & 0 & 0 & 0 \\
0 & 1 & 0 & 0 & 0 & 0 \\
0 & 0 & 1 & L & 0 & 0 \\
0 & 0 & 0 & 1 & 0 & 0 \\
0 & 0 & 0 & 0 & 1 & 0 \\
0 & 0 & 0 & 0 & 0 & 1
\end{array}\right] .
$$

where $\varphi$ is angle between pole face normal direction and beam reference trajectory direction, $\tan \phi_{v} \equiv \tan \varphi-\frac{g}{\rho} \frac{1+\sin ^{2} \varphi}{\cos ^{3} \varphi} I_{2}$ refers to the equivalent vertical focusing angle, $g$ stands for the dipole vertical gap and $I_{2}$ represents a dimensionless integral which is only determined by dipole field distribution [29]. For a dipole with rectangular poles, when the incident beam direction is perpendicular to the pole face, the focusing of the fringe field effect at the incident and the exit of the dipole can be written as $M_{S f}(0)$ and $M_{S f}(\theta)$, where the incident and exit equivalent vertical focusing angles are

$$
\begin{aligned}
\tan \phi_{i} & =-\frac{g}{\rho} I_{2} \\
\tan \phi_{e} & =\tan \theta-\frac{g}{\rho} \frac{1+\sin ^{2} \theta}{\cos ^{3} \theta} I_{2} .
\end{aligned}
$$




\section{APPENDIX B: ELEMENTS IN $M_{A B}$ AND $M_{A C}$}

We define a parameter list as below:

$$
\begin{aligned}
N_{1} & =L_{3}+L_{4} \sec ^{2} \theta+\rho \tan \theta \\
N_{2}(\phi) & =1-\theta \tan \phi \\
N_{3} & =\tan \phi_{i}+\tan \phi_{e}\left(1-\theta \tan \phi_{i}\right) \\
N_{4} & =\rho(1-\cos \theta)+L_{3} \sin \theta .
\end{aligned}
$$

Using the parameters above we can simplify the expressions of all the elements in transport matrices $M_{A B}$ and $M_{A C}$ of both the thin lens and the thick lens; the explicit expressions can be written in Tables III-VI.

TABLE III. Elements of $M_{A B}$ in the thin-lens case.

\begin{tabular}{ll}
\hline \hline Element & \\
\hline$R_{11_{B}}$ & $=\frac{1}{f_{1} f_{2}} \cos \theta\left[L_{2} N_{1}-f_{1}\left(N_{1}-f_{2}\right)-f_{2}\left(L_{2}+N_{1}\right)\right]$ \\
& $=\frac{1}{f_{1} f_{2}} \cos \theta\left\{L_{1}\left[L_{2} N_{1}-f_{2}\left(L_{2}+N_{1}\right)\right]-f_{1}\left[\left(L_{1}+L_{2}\right) N_{1}-f_{2}\left(L_{1}+L_{2}+N_{1}\right)\right]\right\}$ \\
$R_{12_{B}}$ & $=\kappa\left(\rho-\rho \cos \theta+L_{4} \tan \theta\right)$ \\
$R_{13_{B}}$ & $=\rho-\rho \cos \theta+L_{4} \tan \theta$ \\
$R_{16_{B}}$ & $=\frac{1}{f_{1} f_{2}} \sec \theta\left(L_{2}-f_{1}-f_{2}\right)$ \\
$R_{21_{B}}$ & $=\frac{1}{f_{1} f_{2}} \sec \theta\left[f_{1}\left(f_{2}-L_{1}-L_{2}\right)-L_{1}\left(f_{2}-L_{2}\right)\right]$ \\
$R_{22_{B}}$ & $=\kappa \tan \theta$ \\
$R_{23_{B}}$ & $=\tan \theta$ \\
$R_{26_{B}}$ & $=\frac{1}{\rho f_{1} f_{2}}\left\{\rho\left[\theta \rho+L_{4} N_{2}\left(\phi_{e}\right)\right]\left(f_{1}+f_{2}+L_{2}\right)-\left[L_{4} N_{3}-\rho N_{2}\left(\phi_{i}\right)\right]\left(L_{2} L_{3}+f_{1} f_{2}+f_{2} L_{2}+f_{1} L_{3}+f_{2} L_{3}\right)\right\}$ \\
$R_{33_{B}}$ & $=\frac{1}{\rho f_{1} f_{2}}\left\{\rho\left[L_{1}\left(f_{2}+L_{2}\right)+f_{1}\left(L_{1}+L_{2}\right)+f_{1} f_{2}\right]\left[\theta \rho+L_{4} N_{2}\left(\phi_{e}\right)\right]\right.$ \\
$R_{34_{B}}$ & $\left.+\left[f_{1}\left(L_{1}+L_{2}\right)\left(f_{2}+L_{3}\right)+L_{1}\left[L_{2} L_{3}+f_{2}\left(L_{2}+L_{3}\right)\right]+f_{1} f_{2} L_{3}\right]\left[-L_{4} N_{3}+\rho N_{2}\left(\phi_{i}\right)\right]\right\}$ \\
$=$ & $\kappa R_{34_{B}}$ \\
$R_{35_{B}}$ & $=-\frac{1}{\rho f_{1} f_{2}}\left\{\left[L_{3} N_{3}-\rho N_{2}\left(\phi_{e}\right)\right]\left(L_{2}+f_{1}\right)+N_{3} f_{2}\left(f_{1}+L_{2}+L_{3}\right)-f_{2} \rho N_{2}\left(\phi_{e}\right)\right\}$ \\
$R_{43_{B}}$ & $\left.=\frac{1}{\rho f_{1} f_{2}}\left\{\rho N_{2}\left(\phi_{e}\right)-L_{3} N_{3}\right]\left[L_{1} L_{2}+f_{1}\left(L_{1}+L_{2}\right)+f_{2}\left(f_{1}+L_{1}\right)\right]-N_{3} f_{2}\left[f_{1}\left(L_{1}+L_{2}\right)+L_{1} L_{2}\right]\right\}$ \\
$R_{44_{B}}$ & $=\kappa R_{44_{B}}$ \\
$R_{45_{B}}$ & $=-\frac{1}{f_{1} f_{2}}\left[N_{4}\left(-f_{2}+L_{2}-f_{1}\right)+f_{1} f_{2} \sin \theta-f_{2} L_{2} \sin \theta\right]$ \\
$R_{51_{B}}$ & $=-\frac{1}{f_{1} f_{2}}\left\{N_{4}\left[L_{1}\left(L_{2}-f_{2}\right)-f_{1}\left(L_{1}+L_{2}\right)+f_{1} f_{2}\right]-\sin \theta f_{2}\left[L_{1} L_{2}-f_{1}\left(L_{1}+L_{2}\right)\right]\right\}$ \\
$R_{52_{B}}$ & $=\kappa \rho(-\theta+\sin \theta)$ \\
$R_{53_{B}}$ & $=\rho(-\theta+\sin \theta)$ \\
$R_{56_{B}}$ &
\end{tabular}

TABLE IV. Elements of $M_{A C}$ in the thin-lens case.

\begin{tabular}{ll}
\hline \hline Element & \\
\hline$R_{11_{C}}$ & $=1-\frac{1}{f_{1}} L_{2}-\frac{1}{f_{2}}\left(b+L_{3}+L_{5}\right)+\frac{1}{f_{1} f_{2}}\left(L_{2}-f_{2}\right)\left(b+L_{3}+L 5\right)$ \\
$R_{12_{C}}$ & $=b+L_{1}+L_{2}+L_{3}+L_{5}-\frac{1}{f_{1}} L_{1} L_{2}-\frac{1}{f_{2}}\left[\left(L_{1}+L_{2}\right)\left(b+L_{3}+L_{5}\right)\right]+\frac{1}{f_{1} f_{2}} L_{1}\left(L_{2}-f_{2}\right)\left(b+L_{3}+L_{5}\right)$ \\
$R_{21_{C}}$ & $=-\frac{1}{f_{1} f_{2}}\left(f_{1}+f_{2}-L_{2}\right)$ \\
$R_{22_{C}}$ & $=\frac{1}{f_{1} f_{2}}\left[f_{1}\left(f_{2}-L_{1}-L_{2}\right)+L_{1}\left(L_{2}-f_{2}\right)\right]$ \\
$R_{33_{C}}$ & $=1+\frac{1}{f_{1}} L_{2}+\frac{1}{f_{2}}\left(b+L_{3}+L_{5}\right)+\frac{1}{f_{1} f_{2}}\left(L_{2}+f_{2}\right)\left(b+L_{3}+L 5\right)$ \\
$R_{34_{C}}$ & $=b+L_{1}+L_{2}+L_{3}+L_{5}+\frac{1}{f_{1}} L_{1} L_{2}+\frac{1}{f_{2}}\left[\left(L_{1}+L_{2}\right)\left(b+L_{3}+L_{5}\right)\right]+\frac{1}{f_{1} f_{2}} L_{1}\left(L_{2}+f_{2}\right)\left(b+L_{3}+L_{5}\right)$ \\
$R_{43_{C}}$ & $=\frac{1}{f_{1} f_{2}}\left(f_{1}+f_{2}+L_{2}\right)$ \\
$R_{44_{C}}$ & $=\frac{1}{f_{1} f_{2}}\left[L_{1}\left(f_{2}+L_{2}\right)+f_{1}\left(f_{2}+L_{1}+L_{2}\right)\right]$ \\
\hline
\end{tabular}


TABLE V. Elements of $M_{A B}$ in the thick-lens case.

\begin{tabular}{ll}
\hline \hline Element & \\
\hline$R_{12_{B}}^{*}$ & $=R_{12_{B}}-\frac{1}{f_{1} f_{2}} \cos \theta L_{T}\left[-L_{2} N_{1}+f_{1}\left(N_{1}-f_{2}\right)+f_{2}\left(L_{2}+N_{1}\right)\right]$ \\
$R_{14_{B}}^{*}$ & $=\frac{1}{2} \kappa L_{T}\left(\rho-\rho \cos \theta+L_{4} \tan \theta\right)$ \\
$R_{15_{B}}^{*}$ & $=\frac{1}{4} \kappa^{2} L_{T}\left(\rho-\rho \cos \theta+L_{4} \tan \theta\right)$ \\
$R_{22_{B}}^{*}$ & $=R_{22_{B}}-\frac{1}{f_{1} f_{2}} \sec \theta L_{T}\left(f_{1}+f_{2}-L_{2}\right)$ \\
$R_{24_{B}}^{*}$ & $=\frac{1}{2} \kappa L_{T} \tan \theta$ \\
$R_{25_{B}}^{*}$ & $=\frac{1}{2} \kappa^{2} L_{T} \tan \theta$ \\
$R_{34_{B}}^{*}$ & $=R_{34_{B}}+\frac{1}{\rho f_{1} f_{2}} L_{T}\left\{\rho\left(f_{1}+f_{2}+L_{2}\right)\left[\theta \rho+L_{4} N_{2}\left(\phi_{e}\right)\right]+\left[\rho N_{2}\left(\phi_{i}\right)-L_{4} N_{3}\right]\left[L_{2} L_{3}+f_{1}\left(f_{2}+L_{3}\right)+f_{2}\left(L_{2}+L_{3}\right)\right]\right\}$ \\
$R_{35_{B}}^{*}$ & $=R_{35_{B}}+\frac{1}{2} \kappa\left(R_{34_{B}}^{*}-R_{34_{B}}\right)$ \\
$R_{44_{B}}^{*}$ & $=R_{44_{B}}-\frac{1}{\rho f_{1} f_{2}} L_{T}\left\{\left[L_{3} N_{3}-\rho N_{2}\left(\phi_{e}\right)\right]\left(f_{1}+f_{2}+L_{2}\right)+N_{3}\left(f_{1} f_{2}+f_{2} L_{2}\right)\right\}$ \\
$R_{45_{B}}^{*}$ & $=\frac{1}{2} \kappa\left(R_{44_{B}}^{*}-R_{44_{B}}\right)$ \\
$R_{52_{B}}^{*}$ & $=R_{52_{B}}+\frac{1}{f_{1} f_{2}} L_{T}\left[N_{4}\left(f_{1}+f_{2}-L_{2}\right)+f_{2} \sin \theta\left(L_{2}-f_{1}\right)\right]$ \\
$R_{54_{B}}^{*}$ & $=\frac{1}{2} \kappa \rho L_{T}(-\theta+\sin \theta)$ \\
$R_{55_{B}}^{*}$ & $=1+\frac{1}{4} \kappa^{2} \rho L_{T}(-\theta+\sin \theta)$ \\
\hline \hline
\end{tabular}

TABLE VI. Elements of $M_{A C}$ in the thick-lens case.

\begin{tabular}{lc}
\hline \hline Element & Expression \\
\hline$R_{12_{C}}^{*}$ & $b+L_{1}+L_{T}+L_{2}+L_{3}+L_{5}-\frac{1}{f_{1}}\left(L_{1}+L_{T}\right) L_{2}-\frac{1}{f_{2}}\left[\left(L_{1}+L_{T}+L_{2}\right)\left(b+L_{3}+L_{5}\right)\right]$ \\
& $+\frac{1}{f_{1} f_{2}}\left(L_{1}+L_{T}\right)\left(L_{2}-f_{2}\right)\left(b+L_{3}+L_{5}\right)$ \\
$R_{22_{C}}^{*}$ & $=\frac{1}{f_{1} f_{2}}\left[f_{1}\left(f_{2}-L_{1}-L_{T}-L_{2}\right)+\left(L_{1}+L_{T}\right)\left(L_{2}-f_{2}\right)\right]$ \\
$R_{34_{C}}^{*}$ & $b+L_{1}+L_{T}+L_{2}+L_{3}+L_{5}+\frac{1}{f_{1}}\left(L_{1}+L_{T}\right) L_{2}+\frac{1}{f_{2}}\left[\left(L_{1}+L_{T}+L_{2}\right)\left(b+L_{3}+L_{5}\right)\right]$ \\
& $+\frac{1}{f_{1} f_{2}}\left(L_{1}+L_{T}\right)\left(L_{2}+f_{2}\right)\left(b+L_{3}+L_{5}\right)$ \\
$R_{44_{C}}^{*}$ & $=\frac{1}{f_{1} f_{2}}\left[\left(L_{1}+L_{T}\right)\left(f_{2}+L_{2}\right)+f_{1}\left(f_{2}+L_{1}+L_{T}+L_{2}\right)\right]$ \\
\hline \hline
\end{tabular}

[1] F. Pedersen, Beam loading effects in the CERN PS booster, IEEE Trans. Nucl. Sci. 22, 1906 (1975).

[2] W. Gai, A. Kanareykin, A. Kustov, and J. Simpson, Numerical simulations of intense charged-particle beam propagation in a dielectric wake-field accelerator, Phys. Rev. E 55, 3481 (1997).

[3] Z. Chen, Y. Yang, Y. Leng, and R. Yuan, Wakefield measurement using principal component analysis on bunch-by-bunch information during transient state of injection in a storage ring, Phys. Rev. ST Accel. Beams 17, 112803 (2014).

[4] T. Tajima and J. Dawson, Laser Electron Accelerator, Phys. Rev. Lett. 43, 267 (1979).
[5] R. Assmann, R. Bingham, T. Bohl, C. Bracco, B. Buttenschön, A. Butterworth, A. Caldwell, S. Chattopadhyay, S. Cipiccia, E. Feldbaumer et al., Proton-driven plasma wakefield acceleration: a path to the future of high-energy particle physics, Plasma Phys. Controlled Fusion 56, 084013 (2014).

[6] A. Zholents, W. Gai, S. Doran, R. Lindberg, J. Power, N. Strelnikov, Y. Sun, E. Trakhtenberg, I. Vasserman, C. Jing et al., A preliminary design of the collinear dielectric wakefield accelerator, Nucl. Instrum. Methods Phys. Res., Sect. A 829, 190 (2016).

[7] C. Jing, J. Power, A. Zholents et al., Dielectric wakefield accelerator to drive the future FEL light source, Technical 
Report, Argonne National Laboratory (ANL), Argonne, IL, 2011.

[8] K. L. Bane, P. Chen, and P. Wilson, On collinear wakefield acceleration, SLAC Report No. SLAC-PUB 3662, 1985.

[9] H. Figueroa, W. Gai, R. Konecny, J. Norem, A. Ruggiero, P. Schoessow, and J. Simpson, Direct Measurement of Beam-Induced Fields in Accelerating Structures, Phys. Rev. Lett. 60, 2144 (1988).

[10] W. Gai, P. Schoessow, B. Cole, R. Konecny, J. Norem, J. Rosenzweig, and J. Simpson, Experimental Demonstration of Wake-Field Effects in Dielectric Structures, Phys. Rev. Lett. 61, 2756 (1988).

[11] C. Adolphsen, K. Bane, R. Jones, N. Kroll, D. McCormick, R. Miller, M. Ross, T. Slaton, J. Wang, and T. Higo, in Proceedings of the 1999 Particle Accelerator Conference, 1999 (IEEE, New York, 1999), Vol. 5, pp. 3477-3479.

[12] J. G. Power, W. Gai, J. Lewellen, S. Milton, K.-J. Kim, J. Simpson, H. Wang, D. Finley, and H. Carter, in Proceedings of the Particle Accelerator Conference, 2003 (IEEE, New York, 2003), Vol. 3, pp. 1813-1815.

[13] P. B. Wilson, in Proceedings of the 13th SLAC Summer Institute on Particle Physics, SLAC Report No. 296, 1973.

[14] J. Power, W. Gai, and P. Schoessow, Wakefield excitation in multimode structures by a train of electron bunches, Phys. Rev. E 60, 6061 (1999).

[15] C. Jing, A. Kanareykin, J. Power, M. Conde, Z. Yusof, P. Schoessow, and W. Gai, Observation of Enhanced Transformer Ratio in Collinear Wakefield Acceleration, Phys. Rev. Lett. 98, 144801 (2007).

[16] M. Church, Y.-E. Sun, P. Piot, and C. Prokop, Conference Proceedings C1205201, 3009 (2012), http://inspirehep.net/ record/1126862; Report No. FERMILAB-CONF-12-256APC, 2012.J. T. Moody, P. Musumeci, M. S. Gutierrez, J. B. Rosenzweig, and C. M. Scoby, Longitudinal phase space characterization of the blow-out regime of rf photoinjector operation, Phys. Rev. ST Accel. Beams 12, 070704 (2009).

[17] W. Gai, M. Conde, R. Konecny, J. Power, P. Schoessow, J. Simpson, X. Sun, and P. Zou, in Proceedings of the Particle Accelerator Conference, 2001 (IEEE, New York, 2001), Vol. 3, pp. 1880-1882.

[18] C. CLIC, Report No. CERN-2012-007, edited by M. Aicheler, P. Burrows, M. Draper, T. Garvey, P. Lebrun, K. Peach, N. Phinney, H. Schmickler, D. Schulte, and N. Toge, 2012.
[19] C. Jing, S. Antipov, J. Qiu, A. Kanareykin, J. Power, M. Conde, W. Liu, E. Wisniewski, G. Ha, D. Wang et al., The two beam acceleration staging experiment at argonne wakefield accelerator facility, Proceedings, 6th International particle accelerator conference (IPAC 2015): Richmond, Virginia, USA (2015), http://accelconf .web.cern.ch/AccelConf/IPAC2015/papers/wepje020.pdf.

[20] Q. Gao, G. Ha, C. Jing, S. Antipov, J. Power, M. Conde, W. Gai, H. Chen, J. Shi, E. Wisniewski et al., Observation of High Transformer Ratio of Shaped Bunch Generated by an Emittance-Exchange Beam Line, Phys. Rev. Lett. 120, 114801 (2018).

[21] G. Ha, M. Cho, W. Gai, K.-J. Kim, W. Namkung, and J. Power, Perturbation-minimized triangular bunch for high-transformer ratio using a double dogleg emittance exchange beam line, Phys. Rev. Accel. Beams 19, 121301 (2016).

[22] G. Ha, M. Cho, W. Namkung, J. Power, D. Doran, E. Wisniewski, M. Conde, W. Gai, W. Liu, C. Whiteford et al., Precision Control of the Electron Longitudinal Bunch Shape Using an Emittance-Exchange Beam Line, Phys. Rev. Lett. 118, 104801 (2017).

[23] M. De Loos and S. Van der Geer, in the 5th European Particle Accelerator Conference (Barcelona, 1996), p. 1241, http://accelconf.web.cern.ch/accelconf/e96/ PAPERS/THPG/THP001G.PDF.

[24] D. Sagan, in Proceedings of the 10th European Particle Accelerator Conference, Edinburgh, Scotland, 2006 (EPSAG, Edinburgh, Scotland, 2006), pp. 2828-31.

[25] I. V. Bazarov and T. Miyajima, in Proceedings of the 11th European Particle Accelerator Conference, Genoa, 2008 (EPS-AG, Genoa, Italy, 2008), p. 118.

[26] A. Halavanau, G. Qiang, G. Ha, E. Wisniewski, P. Piot, J. Power, and W. Gai, Spatial control of photoemitted electron beams using a microlens-array transverse-shaping technique, Phys. Rev. Accel. Beams 20, 103404 (2017).

[27] S. Baturin, I. Sheinman, A. Altmark, and A. Kanareykin, Transverse operator method for wakefields in a rectangular dielectric loaded accelerating structure, Phys. Rev. ST Accel. Beams 16, 051302 (2013).

[28] J. Shi, H. Chen, C. Tang, S. Zheng, W. Huang, J. G. Power, C. Jing, K.-J. Kim, W. Gai, and D. Li, A 3-cell deflecting RF cavity for emittance exchange experiment at ANL, Nucl. Instrum. Methods Phys. Res., Sect. A 598, 388 (2009).

[29] S. Blitz and R. Molloy, Fringe Field Effects on Bending Magnets, Derived for TRANSPORT/TURTLE, arXiv: 1310.8630. 